\title{
Fungus-mediated synthesis of silver nanoparticles (agnp) and inhibitory effect on aspergillus spp. in combination with antifungal agent
}

\author{
Nevcihan G̈̈RSOY ${ }^{1, *}$ \\ ${ }^{1}$ Sivas Cumhuriyet University, Faculty of Engineering, Department of Food Engineering, Sivas, 58140.
}

\section{Abstract}

In this study, $A$. niger synthesized silver nanoparticules (AgNP) were characterised by using UV-Vis Sphecthrofotometry, Atomic Force Microscopy (AFM) and Transmission Electron Microscope (TEM) Analysis. The antifungal effect of synthesised AgNP and antifungal agent Amphothericin B (Amp-B) combination were investigated against Aspergillus spp. Antimicrobial efficiency were evaluated by Kirby Bauer Agar Disk Diffusion Test. In the end of this study, the particule size of AgNP which biosynthesised on A. niger were measured between 13.2-646.8 nm by AFM. The TEM analysis of AgNPs synthesized on A. niger were determined as a spherical in shape with different sizes $25.5-543.3 \mathrm{~nm}$ in the examined regions. The development of antifungal inhibition zone on A. niger and A. flavus was respectively carried out to evaluate on application of A. niger-AgNP; between 0-0.67 mm, 0-0.42 mm, Amp-B; 0.70-1.50 mm, 0- $0.65 \mathrm{~mm}$, A. niger-AgNP+Amp-B; $1.14-2.00 \mathrm{~mm}, 0-1 \mathrm{~mm}$. According to this study data, antifungal effect of were respectively determined $\% 0.4, \% 1.4$, $\% 2.4 ; \% 0.1, \% 0.45, \% 0.65$ on $A$. niger and $A$. flavus. The antifungal inhibition zone occurrence indicated depent of both fungi results, the Amp-B were increase $\% 43.91$ of $A$. niger-AgNP; A. niger-AgNP+Amp-B \% 40.84 of Amp-B, A. niger-AgNP+Amp-B\% 84.75 of A. niger-AgNP. The statistical evaluation of this study showed that multiple comparison of three application on $A$. niger and A. flavus were significant $(\mathrm{p}<0,005)$.
\end{abstract}

\section{Article info}

History:

Received:01.12.2019

Accepted:03.02.2020

Keywords:

A. niger,

A. flavus,

AgNPs,

Amp-B,

Antimicrobial.

\section{Introduction}

Mycotoxins are a group of toxic chemical secondary metabolites produced by some fungal species such as Aspergillus spp., Fusarium spp., and Penicillium spp. when they grow under favourable conditions on commonly foods and feeds $[1,2,3]$.

Contamination of agricultural products is thought to be caused by infections in which toxin-producing fungi play a role. Mycotoxins generally cause acute and chronic effects on humans and animals. Therefore, they significantly affect human health, food safety and trade. In recent years, pathogenic bacteria and fungi have begun to develop resistance to commercially available antimicrobial agents. This has risen to the point of concern and has become a serious problem today $[4,5]$.

Aflatoxins are mycotoxins mainly produced by Aspergillus parasiticus and Aspergillus flavus. These toxins are naturally present in feeds and foods and may show teratogenic, mutagenic and carcinogenic effects. Major naturally occurring AFs produced by aflatoxigenic fungi are known to be $\mathrm{G}_{1}\left(\mathrm{AFG}_{1}\right), \mathrm{G}_{2}$ $\left(\mathrm{AFG}_{2}\right), \mathrm{B}_{1}\left(\mathrm{AFB}_{1}\right)$ and $\mathrm{B}_{2}\left(\mathrm{AFB}_{2}\right)$ [6-8]. The International Agency for Research on Cancer (IARC) announced that AFB1 is highly carcinogenic $[9,10]$.

The biosynthesis of silver nanoparticles by different biomass is rapidly gaining importance due to ease of formation of nanoparticles and eco-friendly applications [11]. (Reference) The reactivity of silver ions is very high. In addition to inhibiting respiratory and metabolic activity by microorganisms, these ions are also highly capable of preventing physical damage caused by these organisms [12]. In addition, it is claimed that silver ions interact with the bacterial DNA after the bacterium enters the cell, thereby preventing it from proliferating within the cell [13].

Nowadays, both bacteria and fungi have been found to produce silver nanoparticles. Fungi are known to exhibit a very high tolerance to metals and accumulate them quite effectively [14]. Therefore, researchers have begun to focus on these microorganisms in the biological production of metallic nanoparticles [15]. The fungi are extremely efficient producer of extracellular enzymes, due to possible to easily obtain 
production of enzymes. Producing metallic nanoparticles through fungi is a highly eco-friendly approach. This type of production also provides ease of use of biomass and economic viability. Numerous fungal species have been reported to date for the synthesis of AgNPs. These include Rhizopus stolonifer, Penicillium citrinum, Aspergillus favus and Neurospora crassa [16-18].

In addition to the antimicrobial activities of AgNP, the researchers reported that they have antifungal activity against different fungal species [19-22]. According to the results of some studies, Aspergillus terreus is a highly capable organism for the production of silver nanoparticles [23-26]. Researchers claim that AgNPs used at low concentrations inhibit fungal growth but do not have any toxic effect on human cells [27].

Amphotericin B (AmpB), produced by Streptomyces nodosus, a polyen antibiotic, is one of the most potent antifungal compounds currently used, although it has serious side effects [28,29]. AgNPs have been shown to have strong antifungal activity against Candida albicans, Trichosporon beigelii and Trichophyton mentagrophytes when compared with commonly used antifungal agents such as fluconazole and AmpB [30].

In this study, A.niger was selected for the biosynthesis of AgNPs due to its easy isolation, growth on simple medium and has stable biochemical characteristics. AgNPs produced by using cell-free filtrate from $A$. niger, antifungal agent Amp-B alone and in AgNPAmp-B combination against toxigenic Aspergillus species. Additive effects were respectively observed A.niger and A.flavus.

\section{Materials and Methods}

\subsection{Fungi}

A. niger and A. flavus were obtained from Sivas Cumhuriyet University (SCU) Engineering Faculty, Food Enginering Department, Microbiology and Nanofood Technology Lab., Sivas. Cultures were grown in potato dextrose agar (PDA) were incubated for 7 days at $25^{\circ} \mathrm{C}$.

Identification of Aspergillus species is based on microscopic investigations and morphological characteristics of the colonies they produce. Aspergillus strains were purified through single spore isolation. The single conidial isolates were maintained on Potato Dextrose Agar (PDA) medium. Morphological features of Aspergillus cultures were evaluated. The major and remarkable macroscopic features used in the identification of species were colony texture, conidia and reverse colour and colony diameter according to the reports published elsewhere [31,32].

\subsection{Chemicals}

$\mathrm{AgNO}_{3}$ and Amphotericin B (Amp-B) powder has been provided by Sigma \&Aldrich Company, PDA, Potato Dextrose Broth Agar (PDB) Broth agar from Oxoid Company. All chemical used were of analytical grade and solutions were prepared with deionised water in experiments.

\subsection{Biosynthesis of AgNPs}

Biosynthesis of AgNP on A.niger filtrate was performed acoording to Basavaraja et al [33]. For these experiments $250 \mu \mathrm{l}$ of a suspension containing A. niger $1 \times 10^{6}$ spores $/ \mathrm{ml}$ were added to $250 \mathrm{ml}$ erlenmeyer flasks containing $100 \mathrm{ml}$ of Potato Dextrose Broth (PDB). Following the addition of each solvent, the cultures were shake-agitated at $120 \mathrm{rpm}$ for approximately 5 days. At the end of the incubation period, mycelial biomass was separated by using filtration method. Then, in order to remove the media components completely, it was washed with sterile distilled water. After resuspending in $100 \mathrm{~mL}$ distilled water, it was incubated at $25^{\circ} \mathrm{C}$. The suspension was filtered by using a Whatman filter paper No. 1 after $\mathrm{s}$ 24 hours incubation period. The cell filtrate collected and used further AgNP synthesis. For the synthesis 50 $\mathrm{ml}$ of $1 \mathrm{mM} \mathrm{AgNO}{ }_{3}$ aqueous solution was mixed with $50 \mathrm{ml}$ of $A$. niger cell filtrate in $250 \mathrm{ml}$ erlenmeyer flask and agitated at $120 \mathrm{rpm} 25^{\circ} \mathrm{C}$ for 72 hours in dark. A control that does not contain silver ion (contains only biomass) was also run in parallell to the experiments. All solutions were kept in dark to avoid any photochemical reactions during the experiment.

\subsection{Characterization of AgNPs}

\subsubsection{UV-VIS analysis}

Preliminary detection of A. niger-AgNP was carried out by visual observation of colour change in the cell filtrate after incubation process. The reduction of silver ion was confirmed $1 \mathrm{ml}$ of sample was withdrawn after $24 \mathrm{~h}$ by UV-Vis spectrophotometer.

\subsubsection{Atomic force microscopic (AFM) analysis}

AFM reveals the three dimensional picture to characterised nanoparticles. The silver nanoparticles, which are extracted according to the protocol given above, were visualized by using an AFM. A thin layer film of the sample was prepared on a glass slide by 
dropping $100 \mu \mathrm{L}$ of the sample on the slide and was allowed to dry for $5 \mathrm{~min}$. The slides were then scanned with the AFM.

\subsubsection{Transmission electron microscope (TEM) analysis}

Transmission Electron Microscopy (TEM) was used in microscopic evaluation of particle size and shape properties of A. niger-AgNP. AgNP to be prepared in aqueous suspension, 400 mesh carbon film coated copper grids after being removed with aqueous part evaporator, was studied at $120 \mathrm{kV}$.

\subsection{Antifungal activity}

Kirby Bauer Disk Diffusion method was used according to Guatam et al., [34]. The in vitro antifungal activity of AgNPs, Amp-B and AgNP+Amp-B against $A$. niger and $A$. flavus were evaluated on PDA medium. The standard Amp-B disks (Amphotericin B 20;20 $\mu \mathrm{g} /$ disk) were purchased from Oxoid. To determine the combined effect, each standard paper disk was further impregnated with 20 $\mu \mathrm{L}$ of the freshly prepared A. niger-AgNPs. Potato dextrose agar plates were inoculated with a fungal suspension $(20 \mu \mathrm{L})$ of the test fungi. As positive control, standard antifungal Amp-B disks were used, and Amp-B disks impregnated with A. niger-AgNP were placed onto the PDA medium inoculated with test fungi. Fungal cell filtrate, which is used for the synthesis of silver nanoparticles, was used as negative control. The plates were then incubated at $25^{\circ} \mathrm{C}$ for 48 hours. In the case of $\mathrm{A}$. niger-AgNPs, a similar experimental protocol was were applied. The diameters of the zone of inhibition were measued after incubation. The assays were performed in dublicate.

\subsection{Statistical analysis}

The data were subjected to statistical analysis by using SPSS (Ver. 14.00). The antifungal activity were performed the double comparison (A. niger and A. flavus) by Mann Whitney $U$ and comparison in working groups (A. niger-AgNP, Amp-B and A. nigerAgNP+Amp-B) Friedman test.

\section{Results and Discussion}

The strains belonging to the genus Aspergillus Section characteristically present dark-brown to black conidia, with uniseriate or biseriate conidiophores, spherical vesicles and hyaline or lightly pigmented hyphae. A. niger and A.flavus were identified through the examination of their morphological features as per the key descriptions recommended by Raper and Fennel [31] and Klich [32] presented in figure 1.

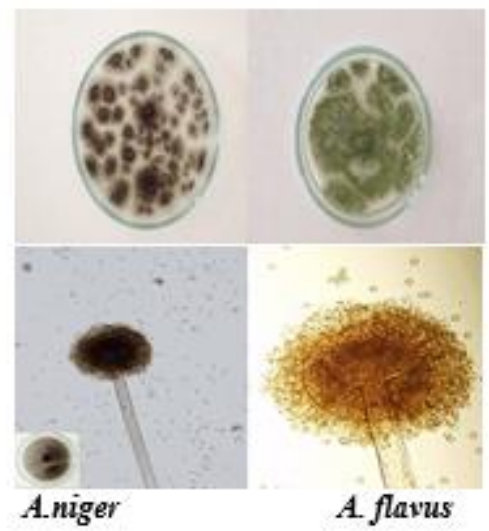

Figure 1. A.niger and A. flavus strain.

The yellow colour of the A. niger fungal cell can clearly be observed before immersion in $\mathrm{AgNO}_{3}$. The colour changed from its natural colour to yellowish brown after $24 \mathrm{~h}$ of incubation as well as of agitation with increasing intensity during the incubation period (Figure 2). The appearance of the brown colour was an indication of the formation of silver nano particles in the medium. Similar colour observations were noted in the several studies [24,25]. Gade et al., [35]. reported that the form of silver ions using A. niger fungus filtrate AgNP thanks to the nitrate reductase and anthraquinone of the fungi.

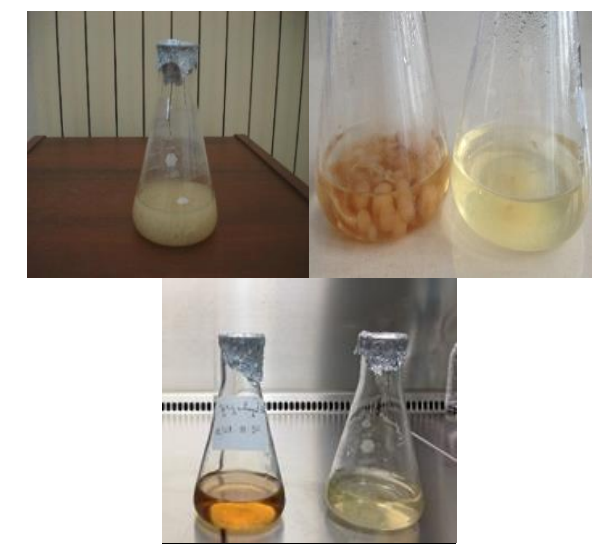

Figure 2. Color changing of fungal filtrate from light yellow to light Brown.

UV-vis spectroscopy is used to record the formation of AgNPs by reduction of $\mathrm{AgNO}_{3}$ by $A$. niger. The optical measurements by UV-Vis spectrophotometer; this analysis showed an absorbance max. peak at $420 \mathrm{~nm}$ which was spesific for the silver nanoparticles (Fig 3). The UV-visible spectroscopy studies could be considered as the most useful technique for structural characterization of silver nanoparticles. The technique outlined above has proved to be very useful for the analysis of nanoparticles [27]. 
It has been found a strong surface plasmon resonance biosynthesized AgNPs at $420 \mathrm{~nm}$. This value confirms the formation of silver nanoparticles. Absorption in the $435-445 \mathrm{~nm}$ range is thought to result from electronic excitation generated by the amino acids tryptophan and tyrosine in the protein $[36,38]$. This absorption value appears to be very close to that exhibited by silver nanoparticles produced by different experimental methods [39-41]. Colloidal silver nanoparticles show strong absorbance between 390 and $420 \mathrm{~nm}$. This is due to mie scattering [40].

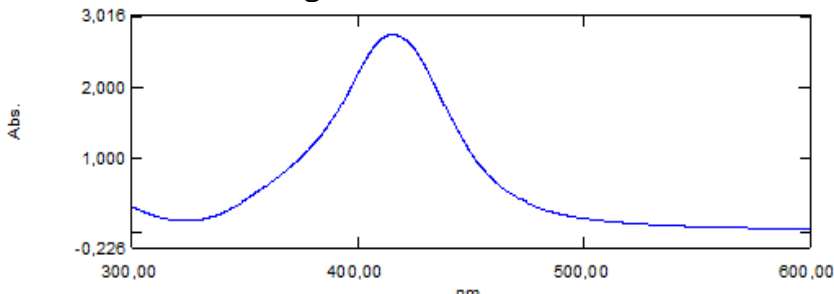

Figure 3. UV-Vis spectrum of A. niger biosynthesized AgNPs.

The mechanism of AgNP synthesis has not yet been fully elucidated. However, it was later proposed that silver ions were necessary for the catalytic activity of NADH-dependent nitrate reductase. This enzyme is secreted into the extracellular environment by the fungus. In line with this hypothesis, the presence of NADH-dependent nitrate reductase enzyme in the extracellular filtrate of fungi tested for the synthesis of nanoparticles was determined. This data is believed to be an important step in validating the hypothesis put forward $[42,43]$.

The sythesized AgNPs were characterized by AFM for its detail size, morphology and agglomeration of silver. It was observed that the silver nanoparticles agglomerated and formed distinct nanoparticles. The particle size of the silver nanoparticles ranges in size from 13.2-646.8 nm (Figure 4). Formation of silver nanoparticles and its agglomeration was clearly observed in figure.
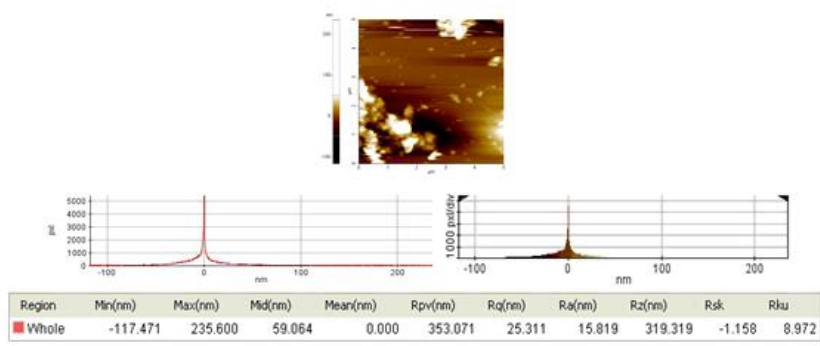

Figure 4. Atomic Force Microscopy result of A. niger synthesized AgNPs.

As a result of TEM analysis of AgNPs synthesized on A. niger, spherical AgNP formations of different sizes between 25.5-543.3 $\mathrm{nm}$ were determined in the examined regions (Figure 5).

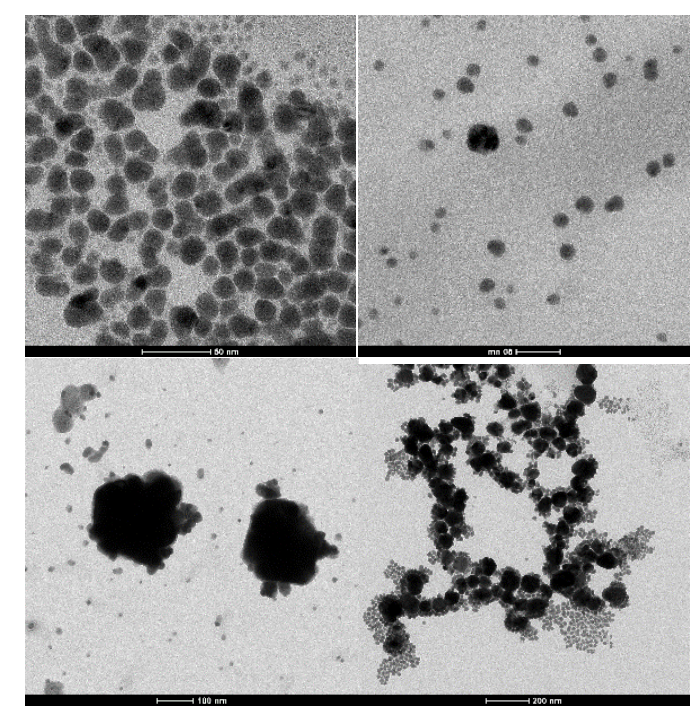

Figure 5. TEM images of $A$. niger synthesized AgNPs in examinated regions.

In the in vitro antifungal activity, Amp- B an antifungal agent that is widely used against many fungal infections, was used as a combination with AgNP and positive (Amp-B) -negative (fungal cell filtrate) control for comparison with AgNP and Amp-B alone. The diameter of inhibition zones and increase in fold area for all the test fungi (A. niger and A. flavus) was measured. However no inhibition zone was obtained in case of the negative control (fungal cell filtrate) (Fig $6)$.

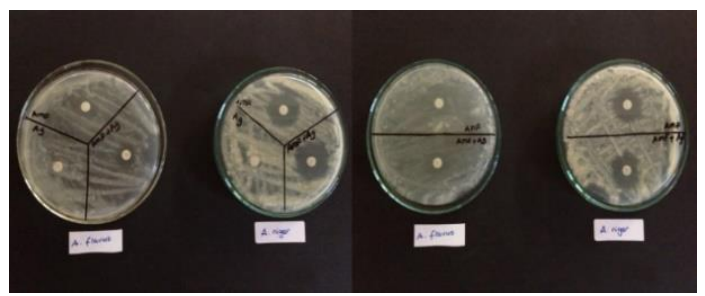

Figure 6. Antifungal activity of A.niger-AgNP, AMP-B against $A$. niger and A. flavus.

The antifungal activity of Amp-B increased significantly in presence of AgNP.

The statistical evaluation of this study showed that multiple comparison of A. niger-AgNP, Amp-B and A.niger and A. flavus were significant $(\mathrm{p}<0,005)$ (Table 1; Figure 7). 
Table 1. Results of statistical analysis of inhibition zones.

\begin{tabular}{llll}
\hline Fungi & Application & $\overline{\mathrm{x}} \pm \mathrm{S}$ & Result \\
\hline A. niger & A.niger-AgNP & $0,33 \pm 1,18$ & $\mathrm{p}=0,0001$ \\
& Amp-B & $0,99 \pm 0,31$ & \\
& A.niger-AgNP+Amp-B & $1,67 \pm 0,27$ & $\chi^{2}=24,00$ \\
A. flavus & A.niger-AgNP & $0,09 \pm 1,13$ & $\mathrm{p}=0,0001$ \\
& Amp-B & $0,31 \pm 0,29$ & \\
& A.niger-AgNP+Amp-B & $0,45 \pm 0,36$ & $\chi^{2}=18,00$ \\
\hline
\end{tabular}
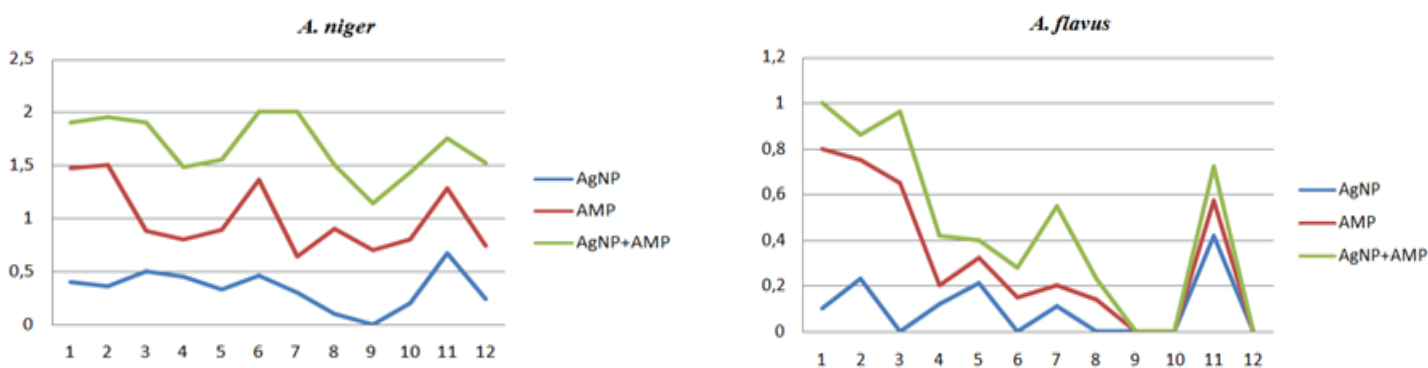

Figure 7. Antifungal effect of A.niger+AgNPs, Amp-B and AgNP+Amp-B on A.niger and A. flavus.

The maximum antifungal activity was observed against $68 \%$ A.niger followed by $31 \%$ A. flavus. This was also shown through the assessment of increases in fold area of activity. It was observed that $A$. niger had the highest increase in the fold area from three aplications (AgNP, Amp-B and AgNP+Amp-B) as respectively $0.67 \mathrm{~mm} ; 1.50 \mathrm{~mm} ; 2 \mathrm{~mm}$ while a much smaller increase in fold area $0,42 \mathrm{~mm} ; 0.70 \mathrm{~mm} ; 1 \mathrm{~mm}$ was observed for A. flavus. These findings collaborate the results obtained by several researcher [44-48]. Noorbakhsh [49], reported that combination of AgNP + Flucanazole and Griseofulvin showed a significant increase as 50\% of AgNPs effect on Trichophyton rubrum. Shahverdi et al [50], investigated that antibacterial effect of $\mathrm{AgNP}$ and $\mathrm{AgNP}+$ Antibiotics combination on Staphylococcus aures and Escherichia coli. They reported thatAgNP+Antibiotics combination increased antimicrobial effect of AgNP.

\section{Conclusions}

As a result, A. niger are among some fungi being used biosynthesis of AgNPs and their antifungal activities. AgNPs can be synthesized with cheapest and ecofriendly methods using A. niger. Moreover, the combination effect of a standard antifungal agent (AMP-B) with $A$. niger biosynthesised $\mathrm{AgNP}$ was observed antifungal effect against $A$. niger whereas much smaller efffect on $A$. flavus. This investigation can be used in the treatment of foods and feeds to reduce the hazards of mycotoxigenic fungi, so more research work especially on experimental needs to be done. In the light of findings is promising the usage of biosynthesized AgNPs has potential as substitutes for food preservatives, pharmaceutical and agrochemical applications.

\section{Acknowledgment}

This study was financially supported by the Scientific Research Council of Sivas Cumhuriyet University (CUBAP M-450).

\section{Conflicts of interest}

The author state that did not have conflict of interests.

\section{References}

[1] Durán N., Marcato P.D., De Souza G.I.H., Alves O.L., and Esposito E., Mechanistic aspects of biosynthesis of silver nanoparticles by several Fusarium oxysporum strains. J. Nanobiotechn. 3 (2005) 34-38.

[2] Brocchi M., Marcato P.D., De Conti R., Gonzaga A.C., Ditondo-Micas A.F., Nakasato G, Alves O.L. and Duran N.A. Comparison of silver nanoparticles produced chemically and by fungal biosynthesis conjugated to clindamycin acting on several bacteria. $J$ Nanosci. Nanotechnol., 21 (2010) 54-61.

[3] CAST. Mycotoxins: Risks in plant, animal and human systems. Council for Agricultural Science and Technology. Ames, Iowa, USA (2003) 139-142. 
[4] Wright G.D. Bacterial resistance to antibiotics: enzymatic degradation and modification. $A d v$. Drug. Deliv. Rev., 57 (2005) 1451-1470.

[5] Wright G.D. Resisting resistance: new chemical strategies for battling superbugs. Chem. Biol., 7 (2000) 127-132.

[6] Sanchez E., Heredia N., Garcia S. Inhibition of growth and mycotoxin production of Aspergillus flavus and Aspergillus parasiticus by extracts of Agave species. Int. J. Food Microbiol., 98 (2005) 271-279.

[7] Razzaghi-Abyaneh M., Shams G.M., Yoshinari T., Rezaee M.B., Jaimand K., Nagasawa H., Inhibitory effects of Satureja hortensis L. essential oil on growth and aflatoxin production by Aspergillus parasiticus. Int. J. Food Microbiol., 123 (2008) 228-233.

[8] Yabe K., Chihaya N., Hatabayashi H., Kito M., Hoshino S., Zeng H. Production of M-/ GMgroup aflatoxins catalyzed by the OrdA enzyme in aflatoxin biosynthesis. Fungal Genet. Biol., 49 (2012) 744-754.

[9] Sidhu O.P., Chandra H., Behl H.M. Occurrence of aflatoxins in mahua (Madhuca indica Gmel.) seeds: synergistic effect of plant extracts on inhibition of Aspergillus flavus growth and aflatoxin production. Food Chem. Toxicol., 47 (2009) 774-777.

[10] Liu S., Qiu F., Kong W., Wei J., Xiao X. Yang M., Development and validation of an accurate and rapid LC-ESI-MS/MS method for the simultaneous quantification of aflatoxin $\mathrm{B}_{1}, \mathrm{~B}_{2}$, $\mathrm{G}_{1}$ and $\mathrm{G}_{2}$ in lotus seeds. Food Control, 29 (2013) 156-161.

[11] Siddiqi K.S., Husen A, Rao R.A.K. A review on biosynthesis of silver nanoparticles and their biocidal properties. J. Nanobiotechnology., 16(1) (2018) 14-21.

[12] Bragg P.D. and D. J. Rannie. The effect of silver ions on the respiratory chain of Escherichia coli. Can. J. Microbiol., 20 (1974) 883-889.

[13] Feng Q.L., Wu J., Chen G.O., Cui F.Z., Kim T.N., and Kim J.O. A mechanistic study of the antibacterial effect of silver ions on Escherichia coli and Staphylococcus aureus. J. Biomed. Mater. Res., 52 (2000) 662-668.

[14] Morkunas I., Woźniak A., Mai V.C., RucińskaSobkowiak R., Jeandet P. The Role of Heavy Metals in Plant Response to Biotic Stress. Molecules. 23(9) (2018) 2320-2328.

[15] Nikolaos P. and Louise E. H. Biological Synthesis of Metallic Nanoparticles by Bacteria, Fungi and Plants. J Nanomed Nanotechnol., 5 (5) (2014) 342-349.
[16] Sastry M., Ahmad A., Khan I., Kumar R. Biosynthesis of metal nanoparticles using fungi and actinomycete. Current Science, 85 (2003)162-170.

[17] Castro-Longoria E., Alfredo R.V.N. and Avalos-Borja M. Biosynthesis of silver, gold and bimetallic nanoparticles using the filamentous fungus Neurospora crassa. Colloids Surf B Biointerfaces, 83 (2011) 42-48.

[18] Honary S., Barabadi H., Gharaei-Fathabad E. and Naghibi F. Green synthesis of silver nanoparticles induced by the fungus Penicillium citrinum. Trop. J. Phar. Res., 12 (2013) 7-11.

[19] Kathiresan K., Manivannan S., Nabeal M.A. and Dhivya B., Studies on silver nanoparticles synthesized by a marine fungus, Pencillium fellutanum isolated from coastal mangrove sediment. Colloids Surf B Biointerfaces, 20 (2009) 133-137.

[20] Bhimba B.V., Gurung S., Nandhini S.U., Silver nanoparticles synthesized from marine fungi Aspergillus oryzae. Int. J. Chem. Tech. Res., 7 (2015) 68-72.

[21] Devi L.S. and Joshi S.R. Ultra structures of silver nanoparticles biosynthesized using endophytic fungi. J. Microsc. Ultrastruct., 3 (2015) 29-37.

[22] Li G., He D., Qian Y., Guan B., Gao S., Cui Y., Yokoyama K., Wang L. Fungus-mediated green synthesis of silver nanoparticles using Aspergillus terreus. Int. J. Mol. Sci., 13 (2012) 466-476.

[23] Abd El-Aziz, A.R., Al-Othman, M.R., Eifan, S.A., Mahmoud, M.A. and Majrashi, M. Green synthesis of silver nanoparticles using Aspergillus terreus (KC462061). Dig. J. Nanomater. Biostruct., 8 (2013) 1215-1225.

[24] Abdel-Hadi A.M., Awad M.F., Abo-Dahab N.F., El-Kady M.F. Extracellular synthesis of silver nanoparticles by Aspergillus terreus: biosynthesis, characterization and biological activity. Biosci. Biotechnol. Res. Asia, 11 (2014) 1179-1186.

[25] Kulkarni P., Rathod V., Hiremath J., Ninganagouda S., Singh D., Singh A.K., Krishnaveni R. Biosynthesis and characterization of silver nanoparticles from Aspergillus terreus and its Antibacterial Efficacy against VRSA Strains. Int. J. Eng. Res. Technol., 3 (2014) 1826-1833.

[26] Rathnayake W.G.I.U., Ismail H., Baharin A., Darsanasiri A.G.N.D., Rajapakse S. Synthesis and characterization of nano silver based natural rubber latex foam for imparting antibacterial and 
anti-fungal properties. Polymer Testing, 31(2012) 586-592.

[27] Ruijgrok E.J., Fens M.H.A.M., BakkerWoudenberg I.A.J.M., Van Etten E.W.M., Vulto A.G. Nebulized amphotericin B combined with intravenous amphotericin B in rats with severe invasive pulmonary aspergillosis. Antimicrob. Ag. Chemother., 50 (2006) 1852-1854.

[28] Rai M., Yadav A., Gade A. Silver nanoparticles as a new generation of antimicrobials Biotechnol. Adv., 27 (2009) 76-83.

[29] Kim K.J., Sung W.S., Moon S.K., Choi J.S., Kim J.G., Lee D.G. Antifungal effect of silver nanoparticles on dermatophytes. J. Microbiol. Biotechnol., 18 (2008)1482-1484.

[30] Kim K.J., Sung W.S., Suh B.K., Moon S.K., Choi J.S., Kim J.G. Antifungal activity and mode of action of silver nanoparticles on Candida albicans. Biometals. 22 (2009) 235242.

[31] Raper K.B. and Fennell D.I. The Genus Aspergillus. Williams and Wilkins, Philadelphia, (1965) 1-686.

[32] Klich M.A. Identification of Common Aspergillus species. Netherlands: Centraalbureau voor Schimmelautures. (2002) 1-116.

[33] Basavaraja S., Balaji S., Lagashetty A., Rajasab A., Venkataraman A. Extracellular biosynthesis of silver nanoparticles using the fungus Fusarium semitectum. Mater. Res. Bull., 43 (2008) 1164-1170.

[34] Gautam V., Singhal L., Arora S., Jha C., Ray P. Reliability of Kirby-Bauer disk diffusion method for detecting carbapenem resistance in Acinetobacter baumanni-calcoaceticus complex isolates. Antimicrob Agents Chemother. 57(4) (2013) 2003-2004.

[35] Gade A., Bonde P., Ingle A., Marcato P., Duran N., Rai M. Exploitation of Aspergillus niger for synthesis of silver nanopar-ticles. Journal of Biobased Material and Bioenergy, 2 (2008) 243247.

[36] Azizi S., Namvar F., Mahdavi M., Bin Ahmad M., Mohamad R. Biosynthesis of Silver Nanoparticles Using Brown Marine Macro alga, Sargassum Muticum Aqueous Extract. Materials (Basel), 6 (2013) 5942-5950.

[37] $\mathrm{Al}$ juraifani A.A.A. and Ghazwani A.A. Biosynthesis of silver nanoparticles by Aspergillus niger, Fusarium oxysporum and Alternaria solani. African Journal of Biotechnology, 14 (2015) 2170-2174.

[38] Singaravelu G., Arockiamary J. S., Ganesh Kumarb V., Govindaraju K. A novel extracellular synthesis of monodisperse gold nanoparticles using marine alga, Sargassum wightii Greville. Colloid Surf B Biointerfaces, 57 (2007) 97-101.

[39] Lee Ch.J., Karim M.R., Vasudevan T., Kim H.J., Raushan K., Jung M.J., Kim D.Y., Lee M.S. A comparison method of silver nanoparticles prepared by the gamma irradiation and in situ reduction methods. Bull. Korea Chem. Soc., 31 (2010) 1993-1996.

[40] Gharibshahi E., Saion E. Influence of dose on particle size and optical properties of colloidal platinum nanoparticles. Int. J. Mol. Sci. 13 (2012) 14723-14741.

[41] Fan X., Zheng W., Singh D. Light scattering and surface plasmons on small spherical particles. Light. Sci. Appl., 3 (2014) 1122-1125.

[42] Roh Y, Bai J.., Lauf R.J, Mcmillan A.D., Phelps T.J., Rawn C.J. Microbial synthesis of metalsubstituted magnetites. Solid State Commun, 118 (2001) 529-534.

[43] Ingle A., Gade A., Pierrat S., Sönnichsen C., Rai M. Mycosynthesis of silver nanoparticles using the fungus Fusarium acuminatum and its activity against some human pathogenic bacteria. Curr. Nanosci., 4 (2008) 141-144.

[44] Anilkumar S., Abyaneh M.K., Gosavi S.W., Kulkarni S.K., Pasricha R., Ahmad A. Nitrate reductase-mediated synthesis of silver nanoparticles from $\mathrm{AgNO}_{3}$. Biotechnol. Lett. 29 (2007) 439-445.

[45] Khan N.T. and Mushtaq M. Determination of Antifungal Activity of Silver Nanoparticles Produced from Aspergillus niger. Biol. Med. (Aligarh), 9 (2016) 363-369.

[46] Kim K.J., Sung W.S., Suh B.K., Moon S.K., Choi J.S., Kim J.G. Antifungal activity and mode of action of silver nano-particles on Candida albicans. Biometals 22 (2009) 235242.

[47] Monteiro D.R., Silva S., Negri M., Gorup L.F., De Camargo E.R., Oliveira R., Barbosa D.B., Henriques M. Antifungal Activity Of Silver Nanoparticles In Combination With Nystatin And Chlorhexidine Digluconate Against Candida albicans. and Candida glabrata Biofilms. Mycoses, 56 (2013) 672-680.

[48] Hassan S.A., Hanif E., Khan U.H., Tanoli A.K. Antifungal activity of silver nanoparticles from Aspergillus niger. Pak. J. Pharm. Sci. 32 (2019) 1163-1166.

[49] Noorbakhsh, F. Antifungal Effects of Silver Nanoparticle alone and with Combination of Antifungal Drug on Dermatophyte Pathogen Trichophyton Rubrum. International 
Conference on Bioscience, Biochemistry and Bioinformatics IPCBEE. IACSIT Press., 5 (2011) 364-367.

[50] Shahverdi A.R., Fakhimi A., Shahverdi H.R., Minanian S. Synthesis and effect of silver nanoparticles on the antibacterial activity of different antibiotics against $S$. aureus and $E$. coli. Nanomedicine, 3 (2007) 168-171. 